\title{
TRFH Domain Is Critical for TRF1-Mediated Telomere Stabilization
}

\author{
Keiji Okamoto and Yoichi Shinkai* \\ Experimental Research Center for Infectious Diseases, Institute for Virus Research, and Graduate School of \\ Biostudies, Kyoto University, 53 Shogoin, Kawara-cho, Sakyo-ku, Kyoto 606-8507, Japan
}

\begin{abstract}
The telomeres are nucleoprotein complexes essential for maintaining the genomic integrity of linear chromosomes. Six telomere localizing proteins form a complex named "shelterin/telosome" to cooperatively regulate telomere length and protect chromosomal ends from DNA damage and repair responses. Mouse embryonic stem (ES) cells lacking TRF1, a shelterin component, exhibit a high-incidence of broken or lost telomere FISH signals, supporting a critical role for TRF1 in telomere maintenance. We demonstrate that these abnormal telomere structures are not caused by the inability of TRF1-deficient cells to recruit TIN2 but are due to a specific role for TRF1 at telomeres. Furthermore, we provide evidence that the mTRF1 TRF homology (TRFH) domain is crucial for this abnormal telomere FISH phenotype. These novel findings suggest that the TRFH domain is crucial not only for dimerization of TRF1 and TIN2-telomere recruitment, but also telomere stabilization.
\end{abstract}

Key words: TRF1/TIN2/FISH/telomere

\section{Introduction}

The telomere is a nucleoprotein complex at the end of linear chromosomes, which is composed of G-rich short nucleotide repeats (e.g. TTAGGG repeats for vertebrates) and associated proteins. The telomere is essential for the maintenance of genomic integrity by regulating telomere length and protecting chromosomal ends from DNA damage and repair responses (Riha et al., 2006; Smogorzewska and de Lange, 2004). There are six telomere localizing proteins in mammals (TRF1, TRF2, TIN2, TPP1, POT1 and RAP1) which form a large complex named "shelterin/telosome" that is critical for telomere function (de Lange, 2005; Liu et $a l ., 2004 \mathrm{a}$ ). One of the shelterin components, telomere repeat binding factor 1 (TRF1), has been identified as a direct telomere double stranded DNA binding protein (Broccoli et al., 1997). TRF1 forms a homodimer through its N-terminal

*To whom correspondence should be addressed: Experimental Research Center for Infectious Diseases, Institute for Virus Research, and Graduate School of Biostudies, Kyoto University, 53 Shogoin, Kawara-cho, Sakyo-ku, Kyoto 606-8507, Japan.

Tel \& Fax: +81-75-751-3991

E-mail: yshinkai@virus.kyoto-u.ac.jp

Abbreviations: TRF1/2; telomere repeat binding factor 1/2, TIN2; TRF1interacting nuclear factor 2, ES cells; embryonic stem cells, FISH; fluorescence in situ hybridization, TRFH domain; TRF homology domain, DAPI; 4',6-diamino-2-phenylindole, ATS; abnormal FISH telomere signals, PNA; peptide nucleic acid.
TRF homology (TRFH) domain and binds to telomeric DNA via the C-terminal Myb domain (Bianchi et al., 1997). TRF1 has been shown to negatively regulate telomere length (Smogorzewska et al., 2000; van Steensel and de Lange, 1997). TRF1-interacting nuclear factor 2 (TIN2) is recruited to the telomere through the TRFH domain of TRF1 (Houghtaling et al., 2004; Kim et al., 1999). Localization of shelterin to telomeres is crippled in mTRF1deficient embryonic stem (ES) cells or by specific knockdown, suggesting a functional role as a scaffold molecule to recruit other shelterin components to telomeres. (Iwano et al., 2004; Liu et al., 2004b; Loayza and de Lange, 2003; Okamoto et al., 2008; Ye et al., 2004a). In our previous studies (Iwano et al., 2004; Okamoto et al., 2008), we reported a high frequency of broken or lost telomere signals by fluorescence in situ hybridization (FISH) analysis in various mTRF1-deficient ES cells, but the nature of these abnormal telomere FISH signals and how TRF1 is involved in this phenotype remained unclear. In this study, we establish a role for the TRFH domain of TRF1 in telomere stabilization. 


\section{Materials and Methods}

\section{Stable transfection of ES cells}

The cDNAs encoding various mTRF1 mutations were subcloned into the vector pCAG-FLAG-IRES-Puro and these plasmids were transfected into mTRF1 conditional knockout (mTRF1 cond-KO) ES cells (Iwano et al., 2004) with Lipofectamine 2000 (Invitrogen, Carlsbad, CA, USA). After $24 \mathrm{~h}$ of transfection, cells were selected with $1 \mu \mathrm{g} / \mathrm{ml}$ puromycin for five days. Multiple independent clones were isolated.

\section{Co-immunoprecipitation}

The previously described protocol for transfection and immunoprecipitation (Ye et al., 2004b) was modified. Briefly, $2 \mu \mathrm{g}$ of each plasmid was transfected into $293 \mathrm{~T}$ cells using TransIT-LT-1 Transfection Reagent (Mirus Bio Corp., Madison, WI, USA). After $48 \mathrm{~h}$, cells were harvested and lysed in lysis buffer $(50 \mathrm{mM}$ Tris- $\mathrm{HCl} \mathrm{pH} 7.5,20 \%$ glycerol, $1 \mathrm{mM}$ EDTA, $150 \mathrm{mM} \mathrm{NaCl}$, $0.5 \%$ TritonX-100, $0.02 \%$ SDS and $1 \mathrm{mM} \mathrm{DTT}$ ) for $5 \mathrm{~min}$ on ice. $\mathrm{NaCl}$ was added to a final concentration of $400 \mathrm{mM}$ and incubated on ice for 5 min followed by the addition of an equal volume of cold-water. Supernatants were collected by immediate centrifugation $(15,000 \mathrm{rpm}$ for $20 \mathrm{~min})$ and immunoprecipitated with $0.5-1 \mu \mathrm{g}$ of anti-MYC antibody (9E10) overnight at $4^{\circ} \mathrm{C}$. Immunoprecipitants were collected by Protein G-Sepharose beads, separated by SDS-PAGE, and subjected to western blot analysis using anti-FLAG (M2, Sigma, St. Louis, MO, USA) and anti-MYC antibodies.

\section{FISH analysis}

Colcemid-treated cells were harvested and incubated in $0.03 \mathrm{mM}$ sodium citrate and fixed with Carnoy fluid (methanol:acetic acid $3: 1$ ) as previously described (Okamoto et al., 2008). Metaphase chromosomes were spread out and fixed with $4 \%$ paraformaldehyde, treated with $1 \mathrm{mg} / \mathrm{ml}$ pepsin, and fixed again. Slides were dehydrated sequentially with $70 \%, 90 \%$, and $100 \%$ ethanol. The Cy3-Telomere peptide nucleic acid (PNA) probe $(0.5 \mathrm{ng} / \mathrm{ml})$ was mounted on the slides, denatured for $3 \mathrm{~min}$ at $80^{\circ} \mathrm{C}$, and incubated at room temperature for $2 \mathrm{~h}$. Slides were washed in Solution I (70\% formamide, $10 \mathrm{mM}$ Tris- $\mathrm{HCl}$ [pH 7.5]) for $15 \mathrm{~min}$, three times with TBS-T for $5 \mathrm{~min}$ and dehydrated again. The cells counterstained with 4',6-diamino-2-phenylindole (DAPI) were analyzed by fluorescence microscopy (Axioplan II, Zeiss, Germany).

\section{Immuno-FISH}

Colcemid-treated cells were spotted onto glass slides by centrifugation and fixed with $4 \%$ paraformaldehyde, as described (Okamoto et al., 2008). Cells were permeabilized with $0.5 \%$ $\mathrm{NP}-40$ and heated in $0.1 \mathrm{M}$ citrate buffer at $120^{\circ} \mathrm{C}$ for $5 \mathrm{~min}$. Preheated Cye3-Tel PNA was hybridized for $2 \mathrm{~h}$ and cells were repermeabilized and incubated with anti-mTIN2 (a kind gift from Dr.
Sahn-Ho Kim) or anti-phospho Histone H2A.X (H2A.XS139ph) (Upstate, now a part of Millipore, Billerica, MA, USA) at $37^{\circ} \mathrm{C}$ for $40 \mathrm{~min}$. Alexa-488 conjugated anti-goat IgG was used as a secondary antibody. The cells counter-stained with DAPI were analyzed by fluorescence microscopy.

\section{Results and Discussion}

TIN2-TRF1 interaction is not required for TRF1-mediated telomere stabilization. In our previous studies, we reported that inactivation of TRF1 in mouse ES cells disrupts the telomeric localization of other shelterin components and induces chromosomal instability, a dysfunctional telomere damage response, growth defects, and abnormal FISH telomere signals (ATS) containing broken or lost telomeres (Iwano et al., 2004; Okamoto et al., 2008). In the latter study, two artificial fusion TRF1 molecules were utilized to rescue these mTRF1-deficient phenotypes. One is the chicken-mouse fusion TRF1 molecule (cmTRF1) (Fig. 2A) which localizes to telomeres but neither binds to mTIN2 nor rescues any of the TRF1-deficient phenotypes. The second fusion, TIN2-cmTRF1, not only tethers TIN2 to telomeres, but also stabilizes other shelterin components at telomeres in the absence of mTRF1. Additionally, TIN2-cmTRF1 suppresses the dysfunctional telomere damage response, chromosomal instability and growth defects of mTRF1-deficient ES cells, although ATS were not rescued (Okamoto et al., 2008). These findings suggest that the induction of the ATS phenotype in mTRF1-deficient ES cells is independent of the telomeric localization of other shelterin components. However, this TIN2 telomere-tethering approach did not completely address whether TIN2-cmTRF1 recapitulates all the functions of endogenous TIN2. In particular, the data of Kim et al. suggest that the TRF1 binding of TIN2 induces conformational changes in TRF1 which enhance TRF1-mediated telomere clustering (Kim et al., 2003). To clarify the relationship between dysfunctional TRF1mediated ATS and TIN2, we applied an alternate approach. Recently, Chen et al. reported that $\mathrm{Phe}^{142}$ (F142) of the human TRF1 TRFH domain is essential for the interaction with human TIN2 (Chen et al., 2008). By aligning human and mouse TRF1, the corresponding amino acid of mTRF1, $\mathrm{F} 129$, was substituted for alanine $\left(\mathrm{mTRF} 1_{\mathrm{F} 129 \mathrm{~A}}\right)$. We tested the ability of the $m T R F 1_{F 129 A}$ mutation for mTIN2 interaction. FLAG-tagged mTRF1 $1_{\mathrm{F} 129 \mathrm{~A}}\left(\mathrm{FLAG}-\mathrm{mTRF} 1_{\mathrm{F} 129 \mathrm{~A}}\right)$ and MYC-tagged mTIN2 (MYC-mTIN2) were transiently expressed in $293 \mathrm{~T}$ cells. FLAG-mTRF1 served as a positive control for mTIN2 interaction. Co-immunoprecipitation experiment using anti-MYC antibody clearly demonstrated that FLAG-mTRF1 was co-immunoprecipitated with mTIN2, but the F129A substitution completely abrogated the binding to mTIN2 (Fig. 1A).

To determine how the F129A mutation affected TRF1 function, we established $m T R F 1$ cond-KO ES cells stably 


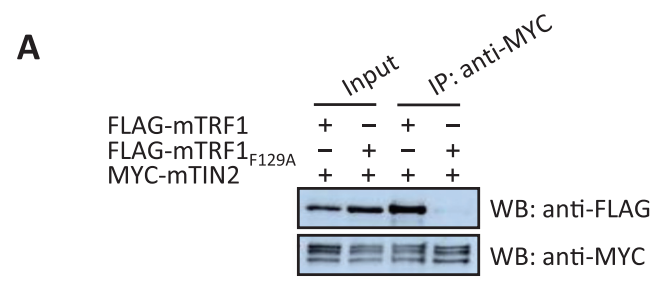

C

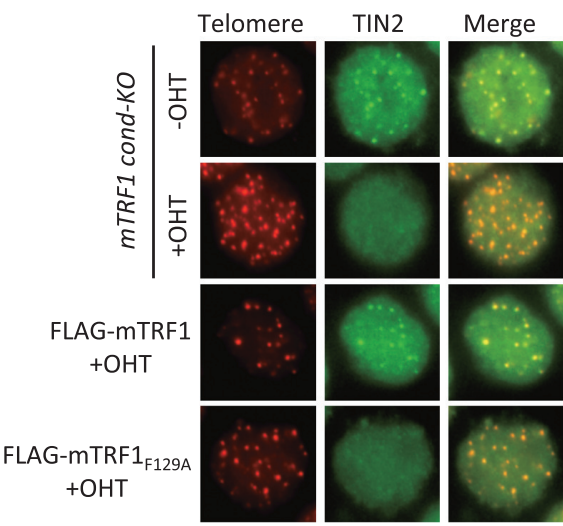

E
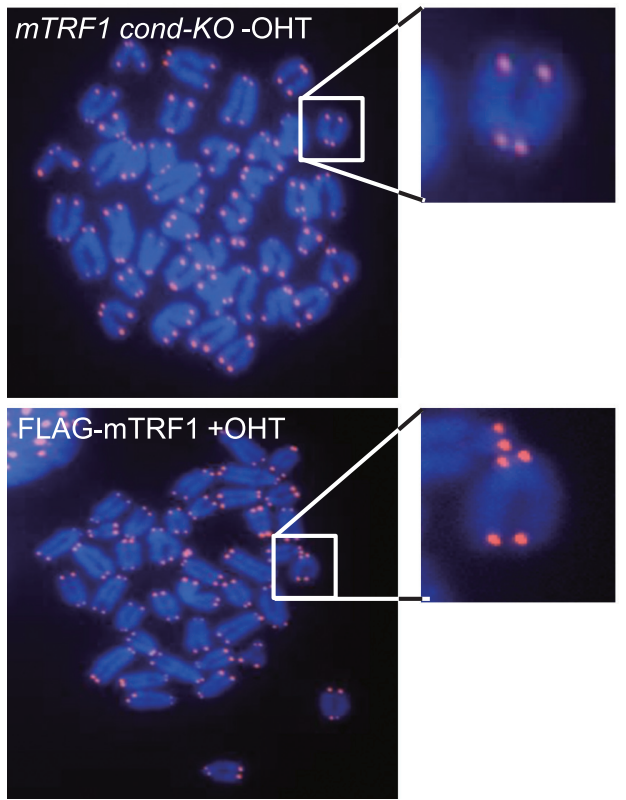

B
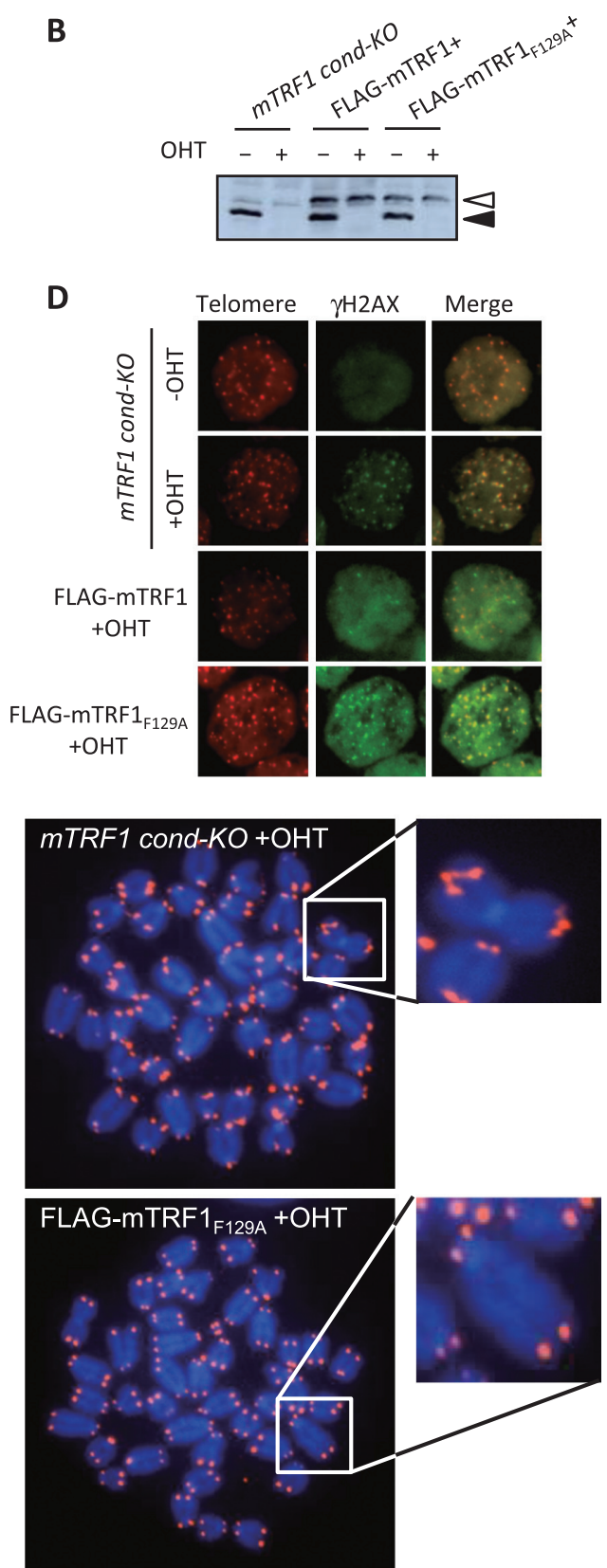

Fig. 1. TIN2-TRF1 association is dispensable for TRF1-mediated telomere stabilization. (A) The association of mTRF1 with mTIN2 is abolished by a point mutation in mTRF1 (mTRF1 $1_{\mathrm{F} 129 \mathrm{~A}}$ ). MYC-tagged mTIN2 (MYC-mTIN2) and either FLAG-tagged mTRF1 (FLAG-mTRF1) or FLAG- mTRF1 $1_{\mathrm{F} 129 \mathrm{~A}}$ were transiently expressed in 293T cells. The transfected cell lysates were subjected to co-immunoprecipitation experiments with anti-MYC (right two

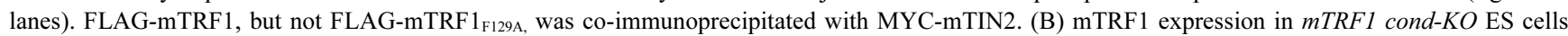
expressing FLAG-mTRF1 (FLAG-mTRF1 $1^{+}$) or FLAG-mTRF1 $1_{\mathrm{F} 129 \mathrm{~A}}\left(\mathrm{FLAG-mTRF} 1_{\mathrm{F} 129 \mathrm{~A}^{+}}\right.$) with or without OHT treatment. The black arrowhead indicates the wildtype mTRF1 which is depleted by OHT treatment and the white arrowhead indicates the FLAG-tagged mTRF1 proteins. mTRF1s were detected by western blotting with anti-TRF1. (C) Telomeric localization analysis of mTIN2 by immuno-FISH. The two fluorescent images (Telomere: red and TIN2: green) for each cell type were superimposed (Merge) to evaluate telomeric localization of mTIN2 (yellow). (D) Telomeric accumulation analysis of $\gamma \mathrm{H} 2 \mathrm{AX}$ by immune-FISH. The two fluorescent images (Telomere: red and $\gamma \mathrm{H} 2 \mathrm{AX}$ : green) for each cell type are superimposed (Merge) to evaluate the accumulation of $\gamma \mathrm{H} 2 \mathrm{AX}$ on telomeres (yellow). (E) Telomere FISH analysis. Metaphase chromosome spreads from each cell type were hybridized with the Cy3$(\mathrm{CCCTAA})_{4}$ PNA probe (red). Each panel represents telomere FISH images of $m T R F 1$ cond-KO ES cells with or without OHT treatment (upper left and right, respectively) or the same cells expressing FLAG-mTRF1 (FLAG-mTRF1+) or FLAG-mTRF $1_{\mathrm{F} 129 \mathrm{~A}}\left(\mathrm{FLAG}-\mathrm{mTRF} 1_{\mathrm{F} 129 \mathrm{~A}}{ }^{+}\right)$with $\mathrm{OHT}$ treatment $($ lower left and right, respectively). The white arrowheads indicate examples of missing or abnormal telomere FISH signals. The small panels show the enlarged image of the chromosomes marked by a white square. 
A

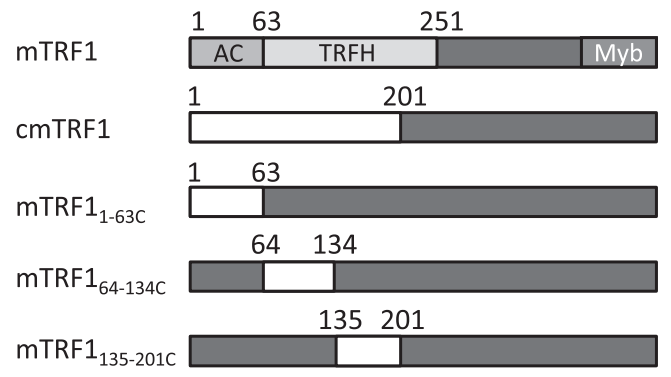

B

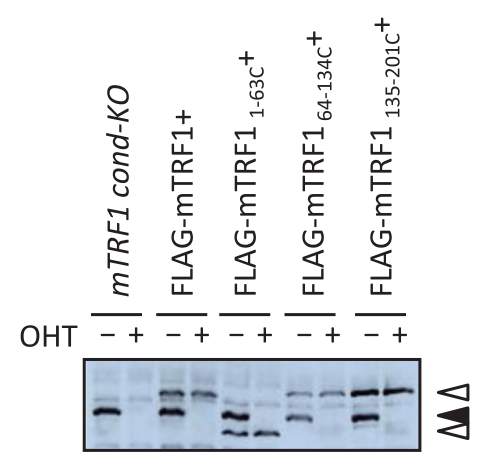

C
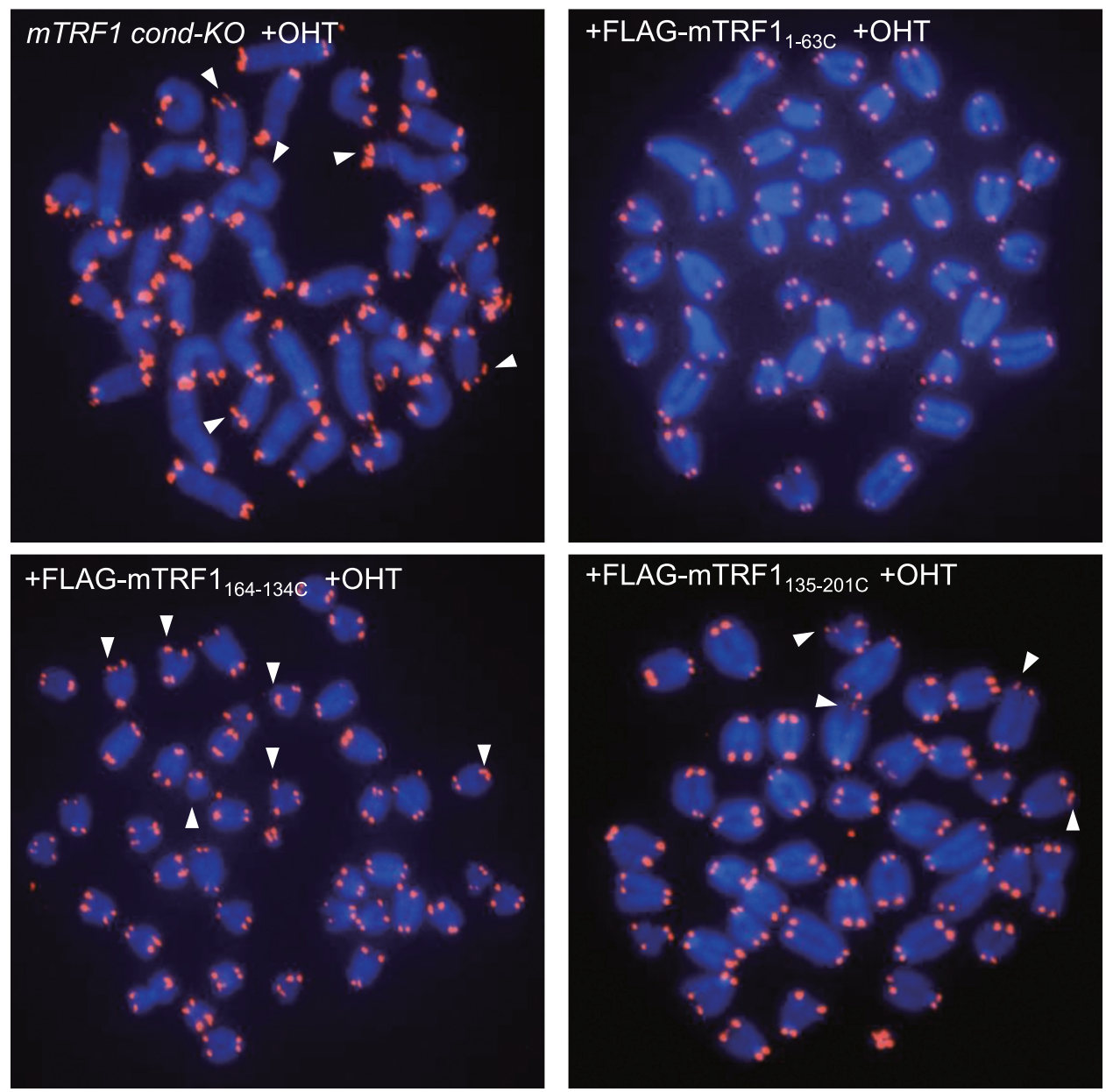

Fig. 2. The TRFH domain of mouse TRF1 is crucial for telomere stabilization. (A) Schematic diagram depicting mTRF1, cmTRF1 and the three newly generated chicken-mouse TRF1 chimeric molecules. The chicken and mouse TRF1 regions are represented in white and black boxes. Ac: acidic domain, TRFH: TRF homology domain, Myb: Myb domain. (B) Protein expression analysis of mTRF1, FLAG-mTRF1 or the various FLAG-cmTRF1 chimeric molecules. Cell lysates of each $m T R F 1$ cond-KO ES cell type with (+) or without (-) OHT treatment were subjected to anti-TRF1 western blot analysis. The black arrowhead indicates the wildtype mTRF1 which is depleted by OHT treatment, and the white arrowhead indicates FLAG-mTRF1 or FLAG-cmTRF1 chimeric molecules. (C) Telomere FISH analysis of mTRF1 cond-KO ES cells or mTRF1 cond-KO ES cells expressing different cmTRF1 chimeric molecules after OHT treatment. The white arrowheads indicate examples of missing or abnormal telomere FISH signals. 
Table I. The SUMMARY OF THE NUMBERS OF ATS

\begin{tabular}{|c|c|c|c|c|c|c|c|c|c|c|c|}
\hline & \multirow{2}{*}{$\begin{array}{c}m T R F 1 \\
\text { cond-KO } \\
(O H T+)\end{array}$} & \multirow{2}{*}{ mTRF1+ } & \multirow{2}{*}{$\begin{array}{c}\text { TIN2- } \\
\text { cmTRF1+ }\end{array}$} & \multicolumn{2}{|c|}{$\mathrm{mTRF} 1_{\mathrm{F} 129 \mathrm{~A}}+$} & \multicolumn{2}{|c|}{$\mathrm{mTRF}_{1-63 \mathrm{C}}+$} & \multicolumn{2}{|c|}{$\mathrm{mTRF} 1_{64-134 \mathrm{C}^{+}}$} & \multicolumn{2}{|c|}{$\mathrm{mTRF}_{135-201 \mathrm{C}^{+}}$} \\
\hline & & & & $\# 1$ & $\# 2$ & $\# 1$ & $\# 2$ & $\# 1$ & $\# 2$ & $\# 1$ & $\# 2$ \\
\hline Number of metaphase spreads & 13 & 11 & 13 & 11 & 12 & 11 & 11 & 12 & 11 & 13 & 12 \\
\hline Ave. number of chromosomes & 39.8 & 41.2 & 40.5 & 42.2 & 42.1 & 40.9 & 41.1 & 40 & 40.8 & 41 & 40.8 \\
\hline Broken telomeres & $\begin{array}{c}503 \\
(24.3)\end{array}$ & $\begin{array}{c}20 \\
(1.1)\end{array}$ & $\begin{array}{c}417 \\
(19.8)\end{array}$ & $\begin{array}{c}39 \\
(2.1)\end{array}$ & $\begin{array}{c}42 \\
(2.1)\end{array}$ & $\begin{array}{c}7 \\
(0.4)\end{array}$ & $\begin{array}{c}24 \\
(1.3)\end{array}$ & $\begin{array}{c}289 \\
(15.1)\end{array}$ & $\begin{array}{c}291 \\
(16.2)\end{array}$ & $\begin{array}{l}150 \\
(7)\end{array}$ & $\begin{array}{c}146 \\
(7.5)\end{array}$ \\
\hline Lost telomeres & $\begin{array}{l}28 \\
(1.4)\end{array}$ & $\begin{array}{c}2 \\
(0.1)\end{array}$ & $\begin{array}{l}35 \\
(1.7)\end{array}$ & $\begin{array}{l}13 \\
(0.7)\end{array}$ & $\begin{array}{c}9 \\
(0.4)\end{array}$ & $\begin{array}{c}0 \\
(0)\end{array}$ & $\begin{array}{c}0 \\
(0)\end{array}$ & $\begin{array}{c}17 \\
(0.9)\end{array}$ & $\begin{array}{l}21 \\
(1.2)\end{array}$ & $\begin{array}{l}10 \\
(0.9)\end{array}$ & $\begin{array}{l}11 \\
(0.6)\end{array}$ \\
\hline
\end{tabular}

The number in the parenthesis indicates the percentage of abnormal telomere signals per all chromosomal ends.

expressing FLAG-mTRF1 or FLAG-mTRF1 $1_{\mathrm{F} 129 \mathrm{~A}}$. The depletion of wildtype mTRF1 with OHT treatment and the expression of exogenous FLAG-mTRF1 or FLAG-mTRF1 $1_{F 129 \mathrm{~A}}$ were confirmed by western blot analysis using anti-mTRF1 (Fig. 1B). In mTRF1 cond-KO ES cells expressing FLAGmTRF $1_{\mathrm{F} 129 \mathrm{~A}}$, telomeric TIN2 foci were remarkably reduced by OHT treatment, similar to the level observed in parental mTRF1 cond-KO ES cells, although mTRF1-deficient cells expressing FLAG-mTRF1 persisted, with telomeric TIN2foci (Fig. 1C). It has been reported that mislocalization of TIN2 from telomeres is coupled with the telomeric accumulation of phosphorylated histone $\mathrm{H} 2 \mathrm{AX}(\gamma \mathrm{H} 2 \mathrm{AX})$ (Okamoto et al., 2008). We confirmed that the loss of TIN2 localization in the mTRF1 cond-KO ES cells expressing FLAGmTRF $1_{\mathrm{F} 129 \mathrm{~A}}$ after OHT treatment correlated with a large accumulation of $\gamma \mathrm{H} 2 \mathrm{AX}$ on telomeres, similar to that observed in mTRF1 cond-KO ES cells with OHT treatment (Fig. 1D).

Finally we examined whether the F129A substitution mutation can suppress the dysfunctional TRF1-mediated ATS. FISH analysis showed that and FLAG-mTRF1 $1_{\mathrm{F} 129 \mathrm{~A}}$ suppressed the ATS induced by mTRF1 depletion similar to those observed in cells expressing FLAG-mTRF1 (Fig. 1E). As summarized in Table I, the frequencies of broken and lost telomere signals per set of examined chromosomal ends for no mTRF1, FLAG-mTRF1+ and FLAG-mTRF1 $1_{\mathrm{F} 129 \mathrm{~A}}+$ cells were $24.3 \%$ and $1.4 \%, 1.1 \%$ and $0.1 \%$, and $2.1 \%$ and $0.4-0.7 \%$, respectively. These results clearly indicate that dysfunctional TRF1-mediated ATS are independent of TIN2 (TIN2-TRF1 binding).

The mTRF1 TRFH domain is crucial for telomere stabilization. Since TIN2-TRF1 binding is dispensable for the ATS phenotype, we sought to identify the compensating portion of the chicken-mouse TRF1 replacement region of cmTRF1. For this purpose, we generated three mTRF1 mutations. In these chimeras, amino acids (aa) 1-63 comprising the acidic domain or aa 64-134 and 135-201 of the TRFH domain were replaced with the corresponding region of cTRF1, aa 1-28, 29-99 or 100-169, respectively (Fig. 2A). Plasmids expressing FLAG-tagged version of these three mutations, FLAG-mTRF $1_{1-63 \mathrm{C}}$, FLAG-mTRF $1_{64-134 \mathrm{C}}$ and FLAG-mTRF $1_{135-201 \mathrm{C}}$ were introduced into $m T R F 1$ cond-KO ES cells and stably expressing clones were established. The expression of each protein was confirmed by western blotting (Fig. 2B). FLAG-mTRF $1_{1-63 \mathrm{C}}$ was detected as a lower molecular weight than the other two mutations and wildtype mTRF1 for an as yet unknown reason. The replacement of the mTRF1 acidic domain with the corresponding region from chicken in $\mathrm{mTRF} 1_{1-63 \mathrm{C}}$ might change the net electric charge of mTRF1, and thus affect protein mobility in SDS-PAGE. FISH analysis of cells expressing these mutations upon OHT treatment clearly showed that mTRF $1_{1-63 \mathrm{C}}$ but not mTRF1 $1_{64-134 \mathrm{C}}$ and mTRF1 $1_{135-201 \mathrm{C}}$ repressed ATS (Fig. 2C). As summarized in Table I, among the cell lines expressing these three mutations, ATS induction was completely suppressed by $\mathrm{mTRF} 1_{1-63 \mathrm{C}}$, and was partially and less efficiently repressed by $\mathrm{mTRF} 1_{135-201 \mathrm{C}}$ and mTRF $1_{64-134 \mathrm{C}}$, respectively (the frequencies of broken and lost telomere signals for $\mathrm{mTRF}_{1-63 \mathrm{C}^{+}}, \mathrm{mTRF}_{135-201 \mathrm{C}}+$ and mTRF $1_{64-134 \mathrm{C}^{+}}+$cells were $0.4-1.3 \%$ and $0 \%, 7-7.5 \%$ and $0.6-0.9 \%$, and $15.1-16.2 \%$ and $0.9-1.2 \%$, respectively). Therefore, we conclude that the mTRF1 TRFH domain, but not the acidic domain causes dysfunctional mTRF1-mediated ATS.

What is the role for the TRFH domain of mTRF1 in telomere stabilization? The TRFH domain, which functions in dimerization, is essential for the function of telomere binding proteins such as TRF1, TRF2 and TAZ1 because all these proteins bind to telomere repeats as homodimers. Our data show that the TRFH domain of mTRF1 has an important additional function at telomeres. One possibility is that the TRFH domain of mTRF1, but not the cTRF1 counterpart, is responsible for the recruitment of critical molecule(s) that control TRF1 function. Indeed, multiple molecules are reported to be associated or co-localized with TRF1 in mammals, such as mitotic checkpoint proteins BubR1 and Mad2, telomerase repressor molecule PIN1X, TRF1-modification proteins CK2 and Fbx4 (Banik and Counter, 2004; Canudas et al., 2007; Chen et al., 2008; Kim et al., 2008; Lee et al., 2006; Muñoz et al., 2009). Fairall et al. suggested that the TRFH domain of TRF1 and TRF2 mediates protein-protein interaction in addition to 
dimerization (Fairall et al., 2001). Indeed, for TRF2 it has been shown that the TRFH domain is required to recruit Apollo to the telomeres. Importantly, the TRF2-mediated recruitment of Apollo is relevant for chromosome end protection (Chen et al., 2008; Lenain et al., 2006; van Overbeek and de Lange, 2006). It is therefore possible that through its TRFH domain, TRF1 also recruits a critical factor, yet to be identified, involved in telomere stabilization. Accordingly, mutations affecting the TRFH domain would produce the ATS phenotype. On the other hand, it remains possible that replacement of the mTRF1 TRFH domain with the chicken version induces a conformational change in mTRF1 which impacts the mTRF1 telomere stabilization function. Additional studies will be required to clarify this issue.

Acknowledgments. We wish to thank Dr. Sahn-Ho Kim (Vattikuti Urology Institute) for providing anti-mTIN2 antibody, Dr. Makoto Tachibana (Kyoto University) for critically reading the manuscript and Lance R. Thomas (Vanderbilt University) for English editing. This work was supported in part by a grant-in-aid from the Ministry of Education, Science, Technology, and Culture of Japan (to Y. S.).

\section{References}

Banik, S.S. and Counter, C.M. 2004. Characterization of interactions between PinX1 and human telomerase subunits hTERT and hTR. $J$. Biol. Chem., 279: 51745-51748.

Bianchi, A., Smith, S., Chong, L., Elias, P., and de Lange, T. 1997. TRF1 is a dimer and bends telomeric DNA. EMBO J., 16: 1785-1794.

Broccoli, D., Smogorzewska, A., Chong, L., and de Lange, T. 1997. Human telomeres contain two distinct Myb-related proteins, TRF1 and TRF2. Nat. Genet., 17: 231-235.

Canudas, S., Houghtaling, B.R., Kim, J.Y., Dynek, J.N., Chang, W.G., and Smith, S. 2007. Protein requirements for sister telomere association in human cells. EMBO J., 26: 4867-4378.

Chen, Y., Yang, Y., van Overbeek, M., Donigian, J.R., Baciu, P., de Lange, T., and Lei, M. 2008. A shared docking motif in TRF1 and TRF2 used for differential recruitment of telomeric proteins. Science, 319: 10921096.

de Lange, T. 2005. Shelterin: the protein complex that shapes and safeguards human telomeres. Genes Dev., 19: 2100-2110.

Fairall, L., Chapman, L., Moss, H., de Lange, T., and Rhodes, D. 2001. Structure of the TRFH dimerization domain of the human telomeric proteins TRF1 and TRF2. Mol. Cell, 8: 351-361.

Houghtaling, B.R., Cuttonaro, L., Chang, W., and Smith, S. 2004. A dynamic molecular link between the telomere length regulator TRF1 and the chromosome end protector TRF2. Curr. Biol., 14: 1621-1631.

Iwano, T., Tachibana, M., Reth, M., and Shinkai, Y. 2004. Importance of TRF1 for functional telomere structure. J. Biol. Chem., 279: 1442-1448.

Kim, M.K., Kang, M.R., Nam, H.W., Bae, Y.S., Kim, Y.S., and Chung, I.K. 2008. Regulation of telomeric-repeat binding factor 1 binding to telomeres by casein kinase 2-mediated phosphorylation. J. Biol. Chem., 283: 14144-14152.

Kim, S., Han, S., You, Y., Chen, D., and Campisi, J. 2003. The human telomere-associated protein TIN2 stimulates interactions between telomeric DNA tracts in vitro. EMBO Rep., 4: 685-691.

Kim, S.H., Kaminker, P., and Campisi, J. 1999. TIN2, a new regulator of telomere length in human cells. Nat. Genet., 23: 405-412.

Lee, T.H., Perrem, K., Harper, J.W., Lu, K.P., and Zhou, X.Z. 2006. The F-box protein FBX4 targets PIN2/TRF1 for ubiquitin-mediated degradation and regulates telomere maintenance. J. Biol. Chem., 281: 759-768.

Lenain, C., Bauwens, S., Amiard, S., Brunori, M., Giraud-Panis, M.J., and Gilson, E. 2006. The Apollo 5' exonuclease functions together with TRF2 to protect telomeres from DNA repair. Curr. Biol., 16: 13031310.

Liu, D., O’Connor, M.S., Qin, J., and Songyang, Z. 2004a. Telosome, a mammalian telomere-associated complex formed by multiple telomeric proteins. J. Biol. Chem., 279: 51338-51342.

Liu, D., Safari, A., O’Connor, M.S., Chan, D.W., Laegeler, A., Qin, J., and Songyang, Z. 2004b. PTOP interacts with POT1 and regulates its localization to telomeres. Nat. Cell Biol., 6: 673-680.

Loayza, D. and De Lange, T. 2003. POT1 as a terminal transducer of TRF1 telomere length control. Nature, 423: 1013-1018.

Muñoz, P., Blanco, R., de Carcer, G., Schoeftner, S., Benetti, R., Flores, J., Malumbres, M., and Blasco, M. 2009. TRF1 controls telomere length and mitotic fidelity in epithelial homeostasis. Mol. Cell. Biol., 29: 16081625.

Okamoto, K., Iwano, T., Tachibana, M., and Shinkai, Y. 2008. Distinct roles of TRF1 in the regulation of telomere structure and lengthening. $J$. Biol. Chem., 283: 23981-23988.

Riha, K., Heacock, M., and Shippen, D. 2006. The role of the nonhomologous end-joining DNA double-strand break repair pathway in telomere biology. Annu. Rev. Genet., 40: 237-277.

Smogorzewska, A. and de Lange, T. 2004. Regulation of telomerase by telomeric proteins. Annu. Rev. Biochem., 73: 177-208.

Smogorzewska, A., van Steensel, B., Bianchi, A., Oelmann, S., Schaefer, M.R., Schnapp, G., and de Lange, T. 2000. Control of human telomere length by TRF1 and TRF2. Mol. Cell. Biol., 20: 1659-1668.

van Overbeek, M. and de Lange, T. 2006. Apollo, an Artemis-related nuclease, interacts with TRF2 and protects human telomeres in S phase. Curr. Biol., 16: 1295-1302..

van Steensel, B. and de Lange, T. 1997. Control of telomere length by the human telomeric protein TRF1. Nature, 385: 740-743.

Ye, J.Z., Donigian, J.R., van Overbeek, M., Loayza, D., Luo, Y., Krutchinsky, A.N., Chait, B.T., and de Lange, T. 2004a. TIN2 binds TRF1 and TRF2 simultaneously and stabilizes the TRF2 complex on telomeres. J. Biol. Chem., 279: 47264-47271.

Ye, J.Z., Hockemeyer, D., Krutchinsky, A.N., Loayza, D., Hooper, S.M., Chait, B.T., and de Lange, T. 2004b. POT1-interacting protein PIP1: a telomere length regulator that recruits POT1 to the TIN2/TRF1 complex. Genes Dev., 18: 1649-1654.

(Received for publication, April 23, 2009, accepted, June 8, 2009 and published online, June 11, 2009) 